\title{
TEST-MODEL CORRELATION OF DRY-FRICTION DAMPING PHENOMENA IN AERO-ENGINES
}

\author{
P. Jean \\ SNECMA, Site de Villaroche, \\ 77550 MOISSY CRAMAYEL, France \\ pierrick.jean@snecma.fr \\ C. Dupont \\ SNECMA, Site de Villaroche, \\ 77550 MOISSY CRAMAYEL, FRANCE
}

\author{
C. Gibert \\ Laboratoire de Tribologie et de Dynamique \\ des Systèmes, UMR 5513 \\ Ecole Centrale de Lyon, \\ 36 avenue Guy de Collongue, 69134 ECULLY \\ CEDEX, France
}

\author{
J.-P. Lombard \\ SNECMA, Site de Villaroche, \\ 77550 MOISSY CRAMAYEL, FRANCE
}

\begin{abstract}
In order to control the risk of high cycle fatigue of bladed disks, it is important to predict precisely the vibration levels and to design damping solutions to attenuate them. Therefore, Snecma has made some efforts in the last years in order to characterize better the damping in aero-engines. Among the various damping sources, friction damping is particularly difficult to model due to its non-linear behaviour [1]. For that purpose, two methods based on multi-harmonic balance strategy have been especially developed for Snecma, dedicated to the study of the non-linear forced response of bladed disks. The first one enables to model the bladed disk equipped with dry-friction dampers [2], and the second one takes into account intrinsic friction located in disk-blade interface [3].

To validate both models experimentally, a test campaign has been carried out in a vacuum chamber on a rotating bladed disk excited by piezoelectric actuators. The blade shanks have been softened in order to increase friction effects. Experimental results show a regular and reproducible behaviour of the non-linear forced response, over various rotation speed and excitation levels. The contributions of friction dampers and friction in blade attachment have been decoupled thanks to glue applied in the blade root.

Both friction phenomena that were observed experimentally at resonance of the blade first bending mode have been reproduced numerically. After updating modeling parameters, an acceptable correlation was found on resonance frequencies, amplitudes and damping levels over the full
\end{abstract}

experimental setup range, which validates these numerical tools for their use in design process.

\section{INTRODUCTION}

The mechanical design of aero-engines bladed disks requires numerical tools to predict the stability and forced response of the blades under aerodynamic loads. Therefore, it is important for an aero-engine designer to evaluate precisely the static and dynamic behaviour of the structure, the aerodynamic loads [4] and the various damping sources (material, aerodynamic and friction). This paper deals with 2 kinds of friction damping phenomena in bladed disks : 1) due to contact in blade-disk attachments, 2) due to friction dampers placed between adjacent blades for vibration attenuation.

Modeling dynamic behaviour of complex 3D structures with friction phenomena is challenging because of the numerical cost of the problem. However, under harmonic loads, the blade forced response is periodic, which enables to resort to Harmonic Balance Methods (HBM), as it has been done in several past studies [5], [6], [7], [8]. Recently, Snecma has integrated in its design process two numerical tools based on HBM: 1) a first tool based on Dynamic Lagrangian Frequency Time method (DLFT) [9], and 2) a second tool based on single- and multiple-point friction dampers models [7]. This paper presents the comparison of the results obtained by these tools and experimental results from a test bench in rotation from Ecole Centrale de Lyon. The comparison between numerical and experimental results is a hard task, due to the non-linearity of friction phenomena and problems of repeatability [9], [10]. In [9], a comparison between DLFT and 
experimental results had been carried out. Here, the experimental campaign has been done again with two major modifications: 1) the blade root has been softened in order to amplify friction phenomena, 2) a series of measurements has been done with the blade bonded to the disk, in order to have a linear reference without friction phenomenon. Therefore, in total, four configurations have been studied:

- bonded dovetail joint without damper : linear reference

- bonded dovetail joint with damper : damper effect only

- free dovetail joint without damper : dovetail joint friction only

- free dovetail joint with damper : combined friction phenomena (not reported here)

First, the experimental setup is described, in which the various configurations are detailed, and the process of tuning is explained. Then, a brief recall of the friction models is given: linear modal analysis with component mode synthesis, DLFT, and friction damper model. Finally the validation of the models by the experimental comparison is carried out. The parameters calibration and updating process is explained, the results are discussed, and some recommendations are addressed.

\section{EXPERIMENTAL SETUP}

\section{Test rig}

The experimental data were obtained with a test facility designed to gain a better understanding of friction phenomena in bladed disks. This test facility based at Ecole Centrale de Lyon has been used for recent studies, such as for the study of high pressure turbine blades with under-platform friction dampers [11].

In the present study, friction zones considered are the dovetail joints between the disk and the blades as well as contact areas between blade platforms and dampers. A view of the test bench used can be seen in Figure 1. The rotating disk supports four blades and is placed in a vacuum chamber so as to minimize the effects of aerodynamic forces. The disk is mounted on a hollow shaft supported by two preloaded contact angle ball bearings and coupled to an electric motor by a drive belt. The blades considered here are compressor blades of an aircraft engine. Two of them are shown in Figure 2. The blades have been remachined in order to make them softer than in [9], for the need of the friction damper study. An underplatform thin-wall dampers $(1.19 \mathrm{~g})$ are shown in Figure 2.

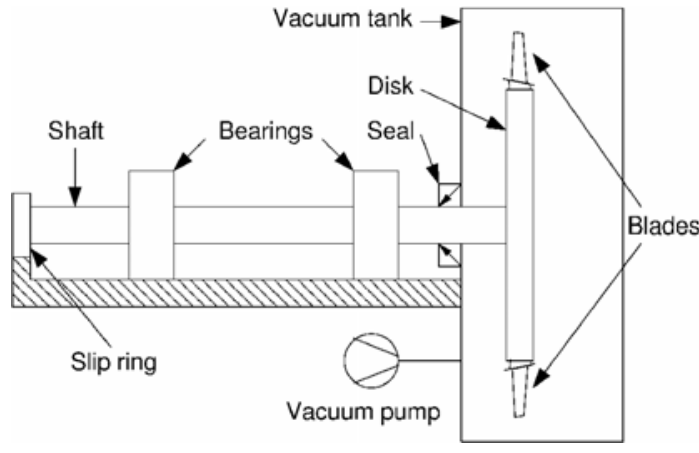

Figure 1: Schematic view of the test bench

\section{Instrumentation}

The four blades are placed around the disk as two diametrically opposite pairs as seen in Figure 1. Two adjacent blades are equipped with piezoelectric actuators and strain gages, while the other two remain bare. Also the disc is instrumented with two strain gages in order to check its participation on the studied resonances.

The two equipped blades, herein called blade 1 and blade 2, are shown in Figure 2. The Lead Zirconate Titanate (PZT) ceramics are $1 \mathrm{~cm}$ square flat layers with a thickness of $1 \mathrm{~mm}$. Care has been taken to have nearly the same positions for actuators and gages on both blades. An insulating epoxy adhesive is used to bond them to the airfoils and to adapt to the slightly curved surface. Two ceramics are used for each blade, one on the pressure side and one on the suction side. A parallel wiring, together with a suitable orientation of polarization directions, allow them to work out of phase and to generate a flexion motion with a common tension signal. The PZT layers are placed on high strain regions of the first bending mode so as to maximize the effectiveness of the excitation. The two blades are equipped similarly with PZT layers so that it is possible to have in phase or out of phase excitations considering the vibration of a pair of blades. A slip ring, located at the extremity of the shaft, is used to bring electrical power to "on-board" electronic devices and PZT exciters and to get measurement signals from rotating part. The strain gages signals are conditioned and converted to current before being transmitted through the shaft and via the slip ring. A Pirani sensor is used to monitor the pressure level inside the vacuum chamber. Actual speed of shaft is measured by means of a tachometer. The vibration level of bearing support is measured by means of piezoelectric accelerometers. Bearing temperatures are monitored by thermocouples.

\section{Measurements}

The first step of the experimental procedure is to pump the air out of the vacuum chamber. The results presented in this paper were obtained with a stabilized pressure of 20 mbar. Then the bladed disk is set into rotation. Five rotational speeds were studied from $1000 \mathrm{rpm}$ to $5000 \mathrm{rpm}$. In order to study the 
frequency response around the modes of interest, a swept sine excitation was provided to the actuators with a sufficiently slow sweep rate so as to avoid artificial distortions of responses in case of low damping levels. For each spinning speed, voltage levels up to $100 \mathrm{~V}$ can be applied to the piezoelectric ceramics. Frequency response functions were provided by measuring only the fundamental component.

As far as the main physical phenomenon involved is friction, checks for repeatability of results was periodically performed during all the test campaign. This one has been performed during several weeks and represents measurement of about 1,000 resonances in different conditions. It was observed that as experiments go on, amplitudes of response tend to decrease while repeating the same test configuration. This is particularly true for tests without damper and dovetails not bonded and at low speed. Cleaning of friction surfaces has shown a benefic restoring effect. The scattering of experimental resonance amplitude has a maximum of $35 \%$. For rotating speed above $3000 \mathrm{rpm}$, the scattering on amplitudes is less than $15 \%$. The frequencies are very reliable as the scattering on this data is less than $0.3 \%$. The frequency shifts due to friction from minimum to maximum excitation level is less than $10 \%$.

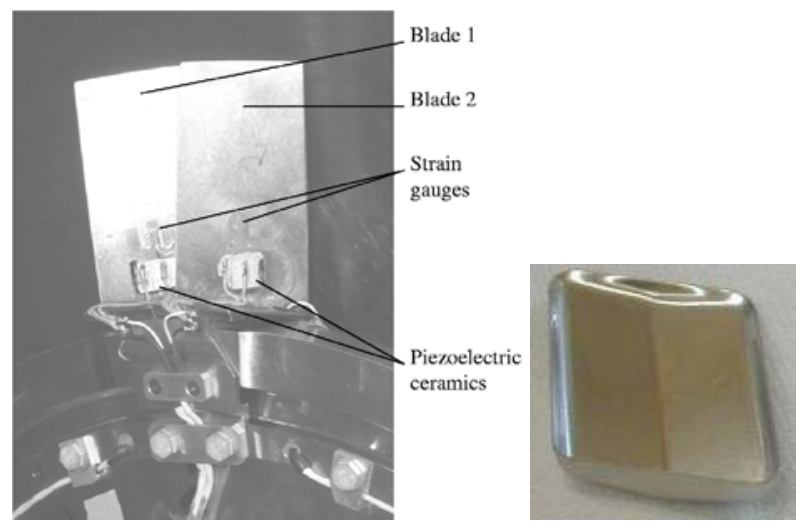

\section{Figure 2 : The two rotating instrumented blades (left) and the thin-wall damper damper (right)}

\section{Holding jaw tests}

As far as dovetail joint are used in the first part of the study, it is common use to test the blades before individually on a holding jaw [9]. In order to quantify the frequency mistuning, the blades were characterised on a rig shown in Figure 3. This is also useful for checking excitation and measurement before mounting blades on the rig but also to establish calibration factors relating strain and tip displacement.

As shown in Figure 4, the same efficiency in term of tension to strain amplitude ratio at resonance of first flap mode for each blade was obtained very satisfactorily. A slight nonlinear effect remains in holding-jaw tests and was very reproducible thanks to the control of tightening torque. The same tightening torque of $10 \mathrm{Nm}$ was imposed in each case to the screw that squeezes the blade root. Except close to the resonance, the linearity of relation between tension level and response was demonstrated.

An important mistuning of about $3 \%$ was noticed between the two blades $1^{\text {st }}$ frequencies as shown in Table 1 . This is unavoidable because of manufacturing tolerances and material discrepancies and maybe because of excitation devices.

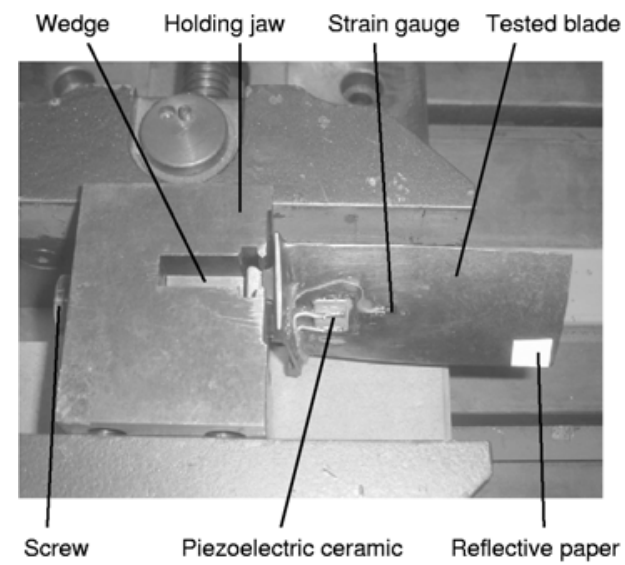

Figure 3 : Holding experimental jaw setup
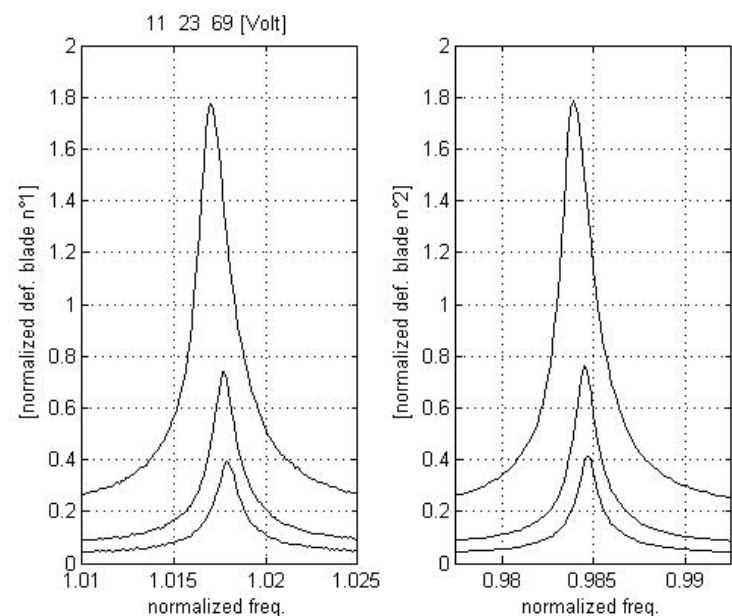

Figure 4 : Holding jaw test. response of blade $n^{\circ} 1$ (left) and $n^{\circ} 2$ (right) with $11,23,69$ volts 
As shown below, this will be compensated by a tuning procedure for the use on the rotating rig resulting in an improvement from previous tests [9].

\begin{tabular}{|c|c|c|c|c|}
\hline & \multicolumn{2}{|c|}{ blade $^{\circ}{ }^{\circ}$} & \multicolumn{2}{c|}{ blade $^{\circ} 2$} \\
$\begin{array}{c}\text { PZT } \\
\text { Voltage } \\
\text { [V] }\end{array}$ & $\begin{array}{c}\text { Resonance } \\
\text { frequency } \\
{[\mathrm{Hz}]}\end{array}$ & $\begin{array}{c}\text { Normalised } \\
\text { damping }\end{array}$ & $\begin{array}{c}\text { Resonance } \\
\text { frequency } \\
{[\mathrm{Hz}]}\end{array}$ & $\begin{array}{c}\text { Normalised } \\
\text { damping }\end{array}$ \\
\hline 12 & 1.0179 & 0.055 & 0.9846 & 0.050 \\
\hline 24 & 1.0176 & 0.059 & 0.9844 & 0.051 \\
\hline 69 & 1.0169 & 0.070 & 0.9839 & 0.069 \\
\hline
\end{tabular}

Table 1 : Holding jaw test, normalised resonance frequency and normalised modal damping ratio of the 1st bending mode

\section{Tuning of rotating blades}

The two blades mounted on the disk and rotating at 2000 rpm have shown 2 resonances at frequencies close to those obtained on holding jaw (Figure 5).

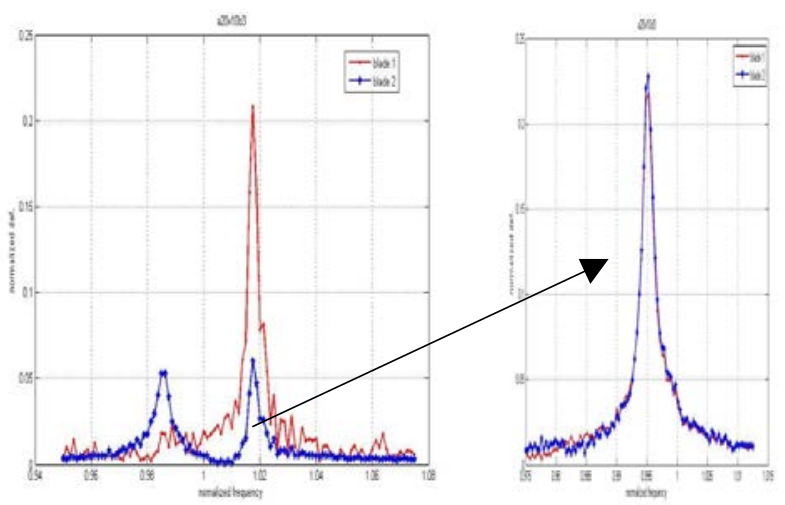

Figure 5 : 2000 rpm, left: before tuning, right : after tuning; red:blade $n^{\circ} 1$, blue: blade $n^{\circ} 2$; out of phase excitation (low level of excitation)

If the two blades were perfectly tuned, there would be an In-Phase (IP) mode where the two blades vibrate in phase, with a strong participation of the disk and an OOP mode where the blades vibrate with equal participation in out-of-phase, with a small participation of the disk. Here, as the blades are mistuned, the IP and OOP modes are disturbed and, in these circumstances, an out-of-phase excitation does not result in a pure out of phase (OOP) motion. Under OOP excitation, blade $\mathrm{n}^{\circ} 1$ has a predominant normalised resonance frequency of 1.017 whereas blade $\mathrm{n}^{\circ} 2$ has two normalised resonance frequencies of 0.985 and 1.017 .
Therefore in order to make experimental results clearer, it was decided to tune the two blades system by adding a small adjustable mass of about $0.5 \mathrm{~g}$. at the tip of the blade $\mathrm{n}^{\circ} 1$. This latter was estimated using a first order sensitivity calculation of $1^{\text {st }}$ flap frequency with respect to the located mass by means of the experimental modal model. Then, the blades were iteratively tested (at a given speed and excitation level) and the mass adjusted until the response of the two blades were equivalent under the OOP excitation. A difference less than 10 $\%$ in amplitude between the two blades was reached in 4 iterations and was considered satisfactory. Interestingly, it was observed that the adding of the mass influences the overall damping. This is due to the fact that the mode shapes of the bladed disk are strongly influenced by the mass. Therefore the friction in the dovetail joint, which is the major contributor in the overall damping (see Figure 6) is also influenced by the mass. For instance, concerning the IP mode, the participation of the disk and therefore the displacement amplitudes in the dovetail joint increase during the tuning process (which is seen on the strain gages signals on the disk), which leads to more dissipation by friction (normalised damping from 0.13 to 0.2 ). On the contrary, during the tuning process, the participation of the disk on the OOP mode decreases together with its modal damping (normalised damping from 0.1 to 0.04). This is considered as an improvement of the experimental setup as the OOP mode is used for testing damper effect.

\section{Bonding of dovetail joints}

The friction effect in dovetail attachment is simultaneous to damper effect and is probably relatively important in the lower half part of the speed-range. Although its influence had been characterized previously, we have tried to suppress it by bonding interfaces between disk and dovetails in order to better isolate the damper effect. The main difficulty was to bear the tangential stress in the interface the mean value of which was estimated of the order of $30 \mathrm{MPa}$ at mid-speed-range. Therefore, we chose for a high performance two parts acrylic/epoxy hybrid adhesive, curable at room temperature and able to meet some other practical requirements. The blades were bonded while rotating at $1000 \mathrm{rpm}$ during about 10 hours for the polymerization to be almost completed. 


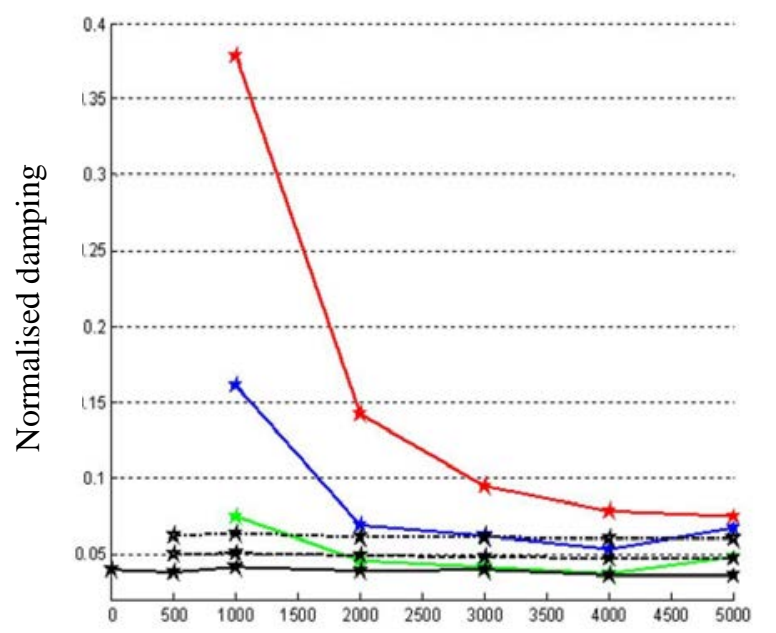

$100 \mathrm{~V}$, free

$40 \mathrm{~V}$, free

$10 \mathrm{~V}$, free

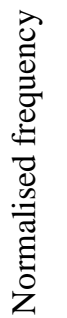

*.-. $100 \mathrm{~V}$, bonded

*-- $40 \mathrm{~V}$, bonded

*... $10 \mathrm{~V}$, bonded

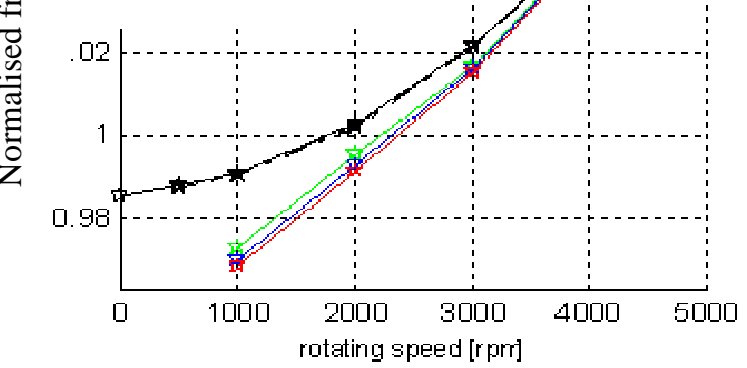

Figure 6 : Comparison of damping ratios and frequencies at several speeds and excitation levels

As shown in Figure 6, the damping ratios obtained with this configuration are quasi constant with respect to rotating speed and always lower compared to the previous case (normalised value of 0.04 to 0.06 ) although a relatively small variation with level remains. The linearity of the dynamic behavior is greatly increased as far as shifts of resonant frequency are less than $-0.1 \%$ when excitation level is multiplied by 10 . One can note a single parabolic curve for the evolution of the first flexural mode frequency obtained at different levels.

At the end of the whole test campaign, degradation of the adhesive layer has been suspected from observed significant changes in responses. This happened only when reaching 5000 rpm with several tests at $100 \mathrm{~V}$ without any damper.

Apart from giving a very pure experimental setup for testing dampers, this configuration brought an answer to [9] where it was wondered if there could be some effect of centrifugal load on damping. The results obtained show that this is not the case, at least for these particular blades and conditions.

\section{NUMERICAL MODELS}

\section{Finite element model}

The first step of friction calculations is a linear modal analysis of the bladed disk in rotation. A numerical model of the bladed disk in cyclic symmetry was created. In order to carry out the preliminary linear calculations, the FE code Samcef was used

The blades and the disk were meshed using second order tetrahedral elements (10 nodes per element), as it can be seen in Figure 7. In order to represent the out-of-phase modes of adjacent blades, an harmonic index, also called nodal diameter, of N/2 is chosen, so that the inter-sector phase angle is equal to $\pi$. The use of cyclic symmerty is necessary in the inhouse friction codes. Indeed, the in-house codes only enable to use 2 super-elements in contact with each other, whereas 5 super-elements would be necessary to model the entire bladed disk. The use of more than 2 super-elements will be available in further developpements.

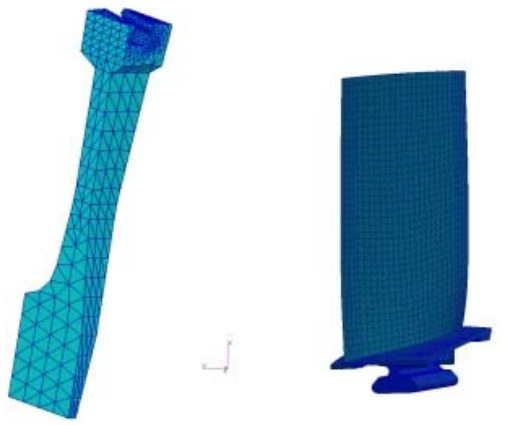

Figure 7 : Bladed disk model in cyclic symmetry

In order to decrease the calculation cost, the first step is to reduce the number of degrees of freedom (dofs), thanks to the Craig \& Bampton component modes synthesis method [12]. A super-element of the disk sector and of the blade is created. The retained nodes are the 64 disk-blade interface nodes, one node for the excitation and two nodes at the tip of the strain gage.

The natural frequencies obtained with the reduced model and those obtained with the full-size model were compared in order to validate the reduced model, as shown in table 2 . The centrifugal forces are taken into account and the frictional contact non-linearity during the static calculation is modeled with node-to-node contact elements. Under this assumption the mass matrix $\mathbf{M}$ and the tangent stiffness matrix $\mathbf{K}_{\mathbf{t}}$ for each substructure are computed and retrieved. The tangent stiffness matrix can be decomposed as

$$
\mathbf{K}_{t}=\mathbf{K}-\mathbf{K}_{c}+\mathbf{K}_{g}
$$


where $\mathbf{K}$ is the structural stiffness, $\mathbf{K}_{c}$ is the centrifugal stiffness (softening effect), and $\mathbf{K}_{g}$ the geometric stiffness matrix which represents the stiffening of the substructure under the rotation-induced stresses. For each substructure, the equations to be solved are written in the reduced basis as

$$
\mathbf{M u ̈}+\mathbf{C} \dot{\mathbf{u}}+\mathbf{K}_{t} \mathbf{u}+\mathbf{f}_{c}=\mathbf{f}_{e x}
$$

where $\mathbf{M}, \mathbf{C}$ and $\mathbf{K}_{\mathbf{t}}$ are mass, material damping and stiffness matrices, and $\mathbf{u}$ is the displacement. $\mathbf{f}_{e x}$ stands for the external forces, $\mathbf{f}_{c}$ represents the contact forces.

\begin{tabular}{|r|r|r|}
\hline $\begin{array}{l}\text { mode } \\
\text { number }\end{array}$ & \multicolumn{1}{c|}{$\begin{array}{c}\text { complete } \\
\text { model }\end{array}$} & \multicolumn{1}{c|}{$\begin{array}{c}\text { condensed } \\
\text { model }\end{array}$} \\
\hline 1 & 1.000 & 1.000 \\
\hline 2 & 3.473 & 3.468 \\
\hline 3 & 3.738 & 3.734 \\
\hline 4 & 5.639 & 5.614 \\
\hline 5 & 8.248 & 8.244 \\
\hline 6 & 9.351 & 9.352 \\
\hline 7 & 13.659 & 13.644 \\
\hline 8 & 14.974 & 14.971 \\
\hline
\end{tabular}

\section{Table 2 Normalised eigenfrequencies of the complete model and the condensed model}

\section{Dovetail joints model}

The model of dovetail joints, called DLFT for Dynamic Lagrangian Frequency Time method, was implemented at Snecma by Ecole Centrale Lyon. It is based on a multiharmonic balance method to compute the forced response of bladed disks with contact and friction nonlinearities in blade roots [8], [9].

Assuming that the steady state motion is periodic, a Galerkin procedure is performed in order to formulate the equations in the frequency domain. Displacements and forces are then represented by multiharmonic vectors which are formed by the Fourier coefficients of the harmonics retained for the resolution. First of all, two exact reductions of the problem are done in the frequency domain : 1) only the degrees of freedom involved in the contact elements are retained, and 2) the problem is written in relative displacements. This leads to the following formulation:

$$
\mathbf{Z}_{r} \tilde{\mathbf{u}}_{r}+\tilde{\bullet}=\tilde{\mathbf{f}}_{r}
$$

where $\tilde{\mathbf{u}}_{r}, \tilde{\bullet}$ and $\tilde{\mathbf{f}}_{r}$ are the multiharmonic vectors of relative displacements, Lagrange multipliers and reduced external forces respectively. $\mathbf{Z}_{r}$ represents the reduced dynamic stiffness matrix. The Lagrange multipliers are equal to the unknown contact forces. A non linear solver is used to determine the zero of the following function:

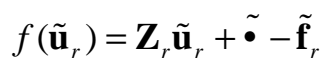

For the computation of function $f, \tilde{\bullet}$ is formulated as a penalization of the equations of motion in the frequency domain:

$$
\tilde{\bullet}=\tilde{\mathbf{f}}_{r}-\mathbf{Z}_{r} \tilde{\mathbf{u}}_{r}+\mathcal{E}\left(\tilde{\mathbf{u}}_{r}-\tilde{\mathbf{x}}_{r}\right)
$$

where $\varepsilon$ is a penalty coefficient and $\tilde{\mathbf{x}}_{r}$ is the vector of relative displacements.

The contact forces are computed in the time domain through a prediction-correction algorithm, where transition criteria between the three possible states (stick, slip and separation) are implemented, which traduces a simple Coulomb friction law. Then, an FFT algorithm is used to get the expression of the contact forces in the frequency domain and the residual $f\left(\tilde{\mathbf{u}}_{r}\right)$ obtained by equation (4) is returned to the non linear solver. Further details of the DLFT algorithm can be found in [9].

\section{Damper model}

A modeling of bladed disk in cyclic symmetry with friction dampers based on a Harmonic Balance Method is used [7]. The principle of modeling is also based on a decomposition of the non-linear dynamics equation on multiple harmonics, and a switch between frequency and time domains is done. More details on the method can be found in [7]. Two versions of the friction damper model have been implemented by Poudou et al [2], we use here the blade-toblade single-point version illustrated in Figure 8, where two dofs per blade are retained in the Craig-Bampton condensation. Adjacent blades dofs are attached through Coulomb element in series with a stiffness element, to account for micro-slipping, and the equations are written in cyclic symmetry. Again, to represent the out-of-phase bending mode, a harmonic index of N/2 is chosen.

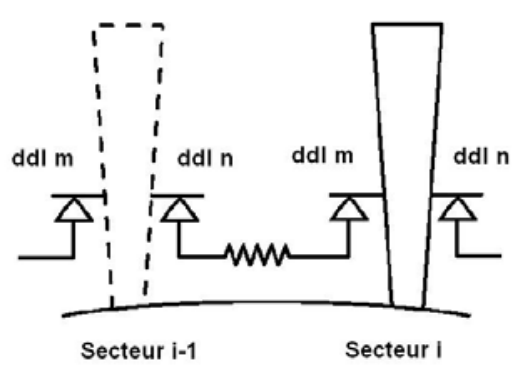

Figure 8 : Damper model in cyclic symmetry 


\section{COMPARISON BETWEEN EXPERIMENTAL AND NUMERICAL RESULTS}

\section{Experimental results}

Figure 9 toFigure 11 show the whole range of experimental results for bonded and free dovetail joints without damper and for free dovetail joint with damper. From these figures, we can make various phenomenological comments.

The bonded dovetail joint configuration is nearly linear as it can be seen in Figure 6 andFigure 9. While Figure 6 shows that the damping ratio is nearly constant over the whole frequency range, the strain amplitude shown in Figure 9 decreases slightly with respect to frequency. In some cases, such as in [11], the piezoelectric actuators can exhibit a nonlinear behaviour, which could explain a decrease of the imposed force. However, here the decrease of amplitude is supposed not to be due to the PZT for 3 reasons. Firstly, eventhough the piezoelectric material used in the present study (PIC151) is the same as in [11], we use here a single-layer actuator bonded directly on the airfoil of the blade rather than a stack fixed bellow the blade platform. In the latter case, an associated seismic mass produces a reaction force to the blade proportionnal to the squared excitation frequency and proportional to the mass and piezo elongation. It is known that the relation between piezo elongation and applied tension can exhibit hysteresis. This has been experimentally tested for exemple in reference [13] using an electrical field up to 2 $\mathrm{kV} / \mathrm{mm}$. As far as we apply here at most $100 \mathrm{~V}$ for a plate with $1 \mathrm{~mm}$ thickness, this ef fect is believed to be of minor importance. Secondly, for a linear oscillator, there is a decrease of amplitude $x$ with respect to the square of the frequency $\omega_{n}$ at the resonance : $x \propto \frac{1}{2 \xi \omega_{n}{ }^{2}}$ as the damping ratio $\xi$ is constant with respect to the frequency. This is confirmed by looking at the product $x \omega_{n}{ }^{2}$ which is nearly constant with respect to $\omega_{n}$. Thirdly, the calibration of the excitation force gives nearly constant values of force over the frequency range, and a linear relation of calibrated with respect to input voltage.

Figure 10 shows the effect of friction damping in the blade root. We can see that slip occurs particularly at low speed. At high speed, the centrifugal forces make the blade-disk interface stuck. We notice however that the amplitudes decrease again over $4000 \mathrm{rpm}$, probably for the same reason than in Figure 9, since the damping ratio tends to a constant value in Figure 6. At $1000 \mathrm{rpm}$, we can clearly see the sensibility of the resonance frequency to the excitation level. As the blade root slips, the resonance frequency shifts in the low frequencies.

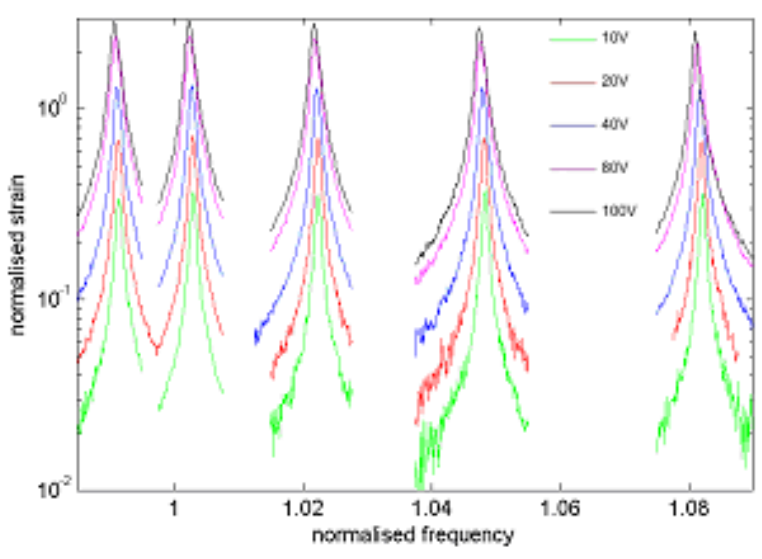

Figure 9 : Experimental results of bonded dovetail joint configuration without damper for various excitation levels $(10,20,40,80,100 \mathrm{~V})$, at various speeds $(1000,2000,3000,4000,5000 \mathrm{rpm})$

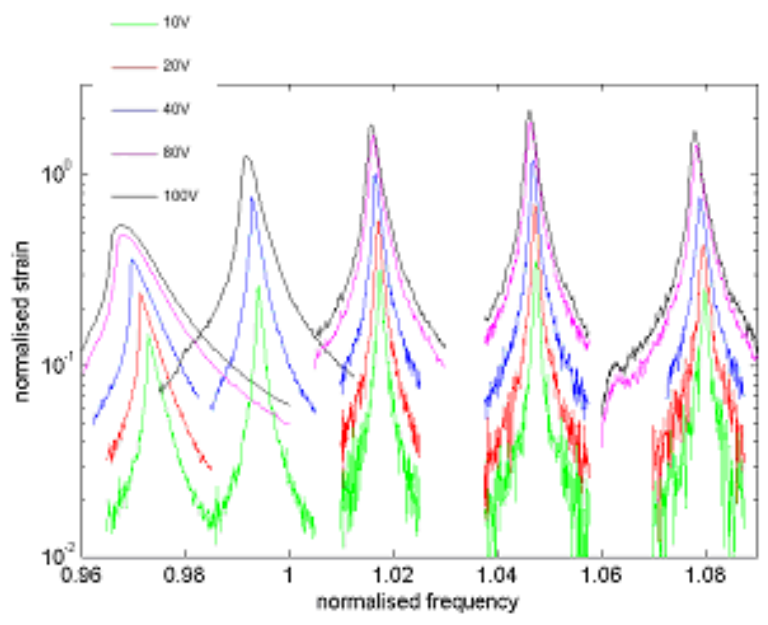

Figure 10 : Experimental results of dovetail joint configuration without damper for various excitation levels $(10,20,40,80,100 \mathrm{~V})$, at various speeds $(1000$, $2000,3000,4000,5000 \mathrm{rpm})$

Moreover, Figure 11 shows the efficiency of the friction damper on the vibration level. It induces mainly a vibration attenuation of $-23 \mathrm{~dB}(-93 \%)$. We can also notice that the attenuation performance depends on the rotation speed. The friction damper is efficient beyond 1000rpm and loses a little performance for high speeds. This phenomenon is wellknown: at a given excitation level, the friction damper passes through 3 states for various centrifugal forces:

totally slip state at low normal forces, therefore low dissipation / high amplitudes

- totally stick state at high normal forces, therefore low dissipation / high amplitudes

stick-slip state at intermediate normal forces, therefore high dissipation / low amplitudes. 
The efficiency of the damper depends on the frequency bandwidth between the slip and the stick states. Here, the damper is particularly efficient and its efficiency is quite robust with frequency because the blade root has been softened to maximise this bandwidth.

Finally, we can make a few comments on resonance frequencies. First, if we compare Figure 9 and Figure 11, we see that the frequencies shift on the right when the friction damper is present, because the damper couples the blades to each other. Secondly, Figure 11 shows the influence of the excitation level on the resonance frequency. When the excitation level increases, the resonance frequency shifts in the low frequencies, as was observed for dovetail joint friction, because the dynamic forces make the interfaces slip.

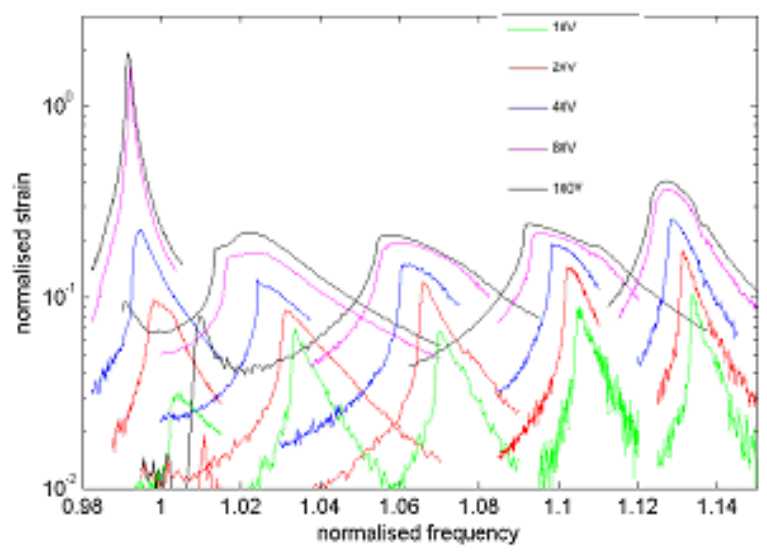

Figure 11: Experimental results of bonded dovetail joint configuration with damper for various excitation levels $(10,20,40,80,100 \mathrm{~V})$, at various speeds (1000, $2000,3000,4000,5000 \mathrm{rpm}$ )

\section{Updating model in linear configuration}

The calibration of the damping matrix $\mathbf{C}$ has been done as follows. The modal damping coefficients implemented are the experimental values from the bonded dovetail joints configuration. In the algorithms used for friction modeling, $\mathbf{C}$ is implemented as a Rayleigh matrix $(\mathrm{C}=\alpha \mathrm{K}+\beta \mathrm{M})$. However, the Rayleigh model is not adapted since the damping ratio is nearly contant with respect to the frequency. Therefore, the values of $\alpha$ and $\beta$ are updated to obtain the adequate damping ratio but for each regime and each excitation level ( $\alpha$ is fixed to zero).

Contrary to what was done in [9], no piezoelectric model of the PZT exciter was done, but a calibrated point force was used. For the bonded dovetail joints configuration, the linear model is calculated as described before, for which the excitation force is calibrated so that numerical and experimental strain amplitudes fit. The obtained calibrated force is nearly constant with respect to the frequency and is nearly linear with respect to the input voltage, which gives confidence about the linearity of the PZT exciter.

Moreover, in the linear calculation, the Young modulus is updated so that order the eigenfrequency of the out-of-phase bending mode is equal to that found experimentally, in the bonded dovetail joints configuration. After the updating process of Young modulus, linear damping and excitation force, simulation results fit very well with experimental results of Figure 9. The resonance frequency fits well for all the studied speeds, as it can be seen in Figure 12. The parabolic evolution of resonance frequency in the bonded case is well captured by the linear model with centrifugal forces.

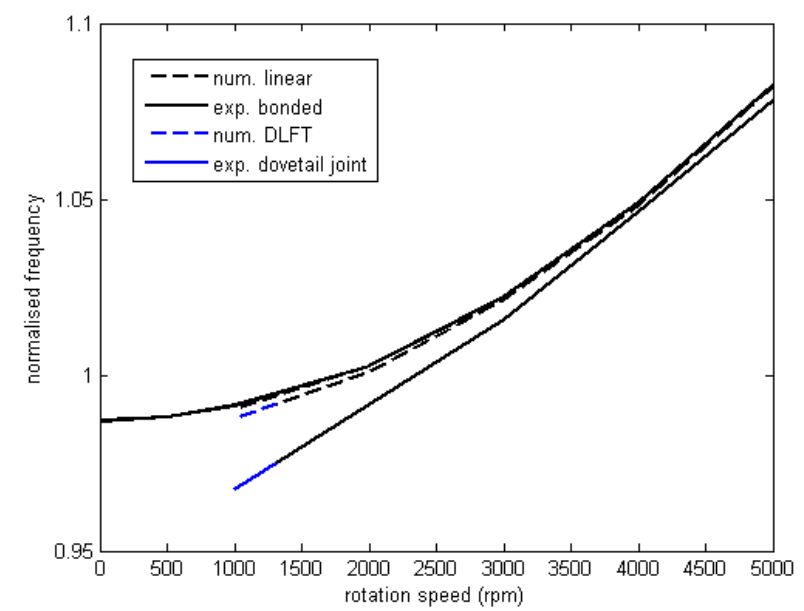

Figure 12 : Comparison between experimental (solid line) and simulation (dashed line) results for bonded (black) and dovetail (blue) joint configurations $($ excitation $=100 \mathrm{~V})$

\section{Dovetail joint friction}

Once the update of linear damping and excitation force is done on the bonded case, the DLFT calculation is applied and compared to experimental results for all the excitation levels and all the rotation speeds.

Figure 13 and Figure 14 show the experimental and simulation strain responses for various excitation levels at $1000 \mathrm{rpm}$. Figure 15 shows the experimental and simulation strain amplitude at resonance. Figure 16 shows the experimental and simulation equivalent damping measured on the strain responses (-3dB bandwidth). To carry out the simulation, the only calibration parameter is the friction coefficient. After a few tests of sensitivity, a fixed friction coefficient of $\mu=0.25$ has been chosen for all speeds and excitation levels, which leads to a quite good precision compared to experimental results while being an acceptable value for steel-steel contact. The decrease of strain amplitude from Figure 9 to Figure 10 is globally well captured by 
simulation, which means that the DLFT method reproduces quite well the friction phenomenon. Note that the results could be slightly improved through a sensitivity analysis and an optimisation process, but $\mu=0.25$ is close to a satisfied value. With such a simple calibration method, we see that the results are quite satisfactory below $4000 \mathrm{rpm}$ in terms of strain levels and damping levels. Below $4000 \mathrm{rpm}$, the gap between experimental and simulation results is $7 \%$ in average for the amplitude, and $18 \%$ in average for the damping. It is to be noticed that the decrease in amplitude at $5000 \mathrm{rpm}$ is not captured by the simulation, but the origin of this decrease is not well understood. It could be another reason than friction.

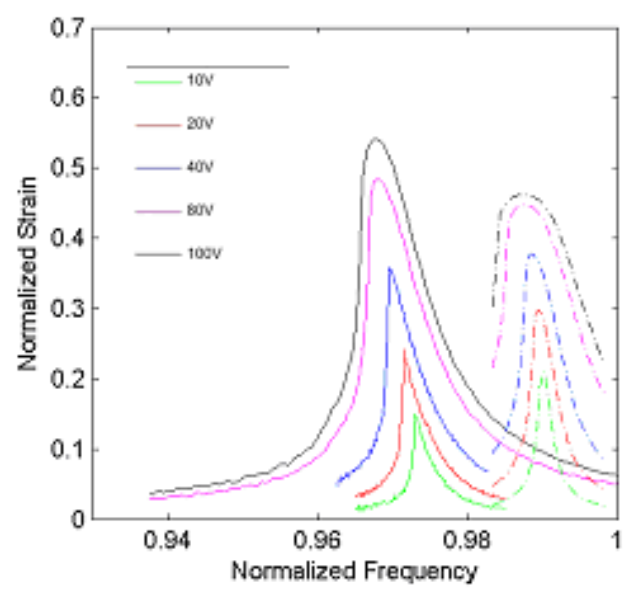

Figure 13 : Normalised experimental (solid line) and numerical (dashed line) strain results for dovetail joint without damper configuration, at $1000 \mathrm{rpm}$

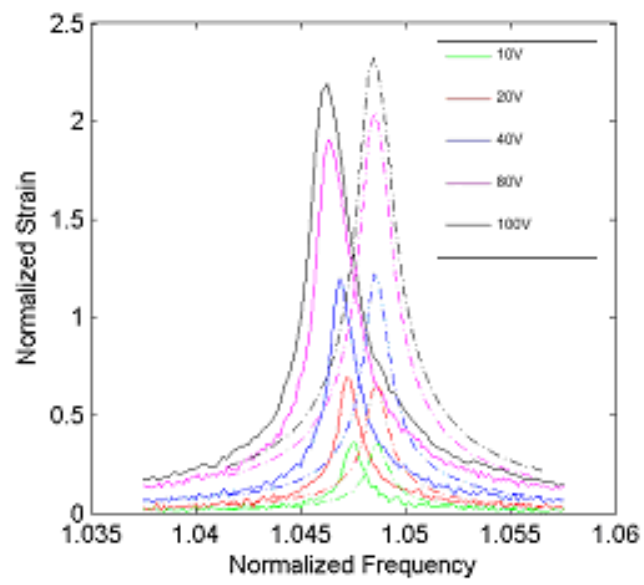

Figure 14 : Normalised experimental (solid line) and numerical (dashed line) strain results dovetail joint without damper configuration, at 4000 rpm

The difference is the highest at $1000 \mathrm{rpm}$, even though the value of $\mu=0.25$ was a good compromise since at low excitation level, simulation is higher than experimental amplitude whereas the hierarchy is inversed at high excitation level. At this speed, we can conclude that the non-linearity of the DLFT seems to be a bit too strong, due to high nonlinearity of the Coulomb friction law. The friction law can be a way of improvement of the method. Finally, concerning resonance frequency, two comments have to be done. First, as it can be seen in Figure 12, the friction in dovetail joint make the resonance frequency shift to the low frequencies, if we compare black and blue curves. However, this shift is much lower in simulation (dashed lines) than in the experiment (solid lines). Secondly, Figure 13 and Figure 14 show the sensitivity of the resonance frequency with respect to the excitation level. Again, the simulation gives a lower sensitivity than the experiment.

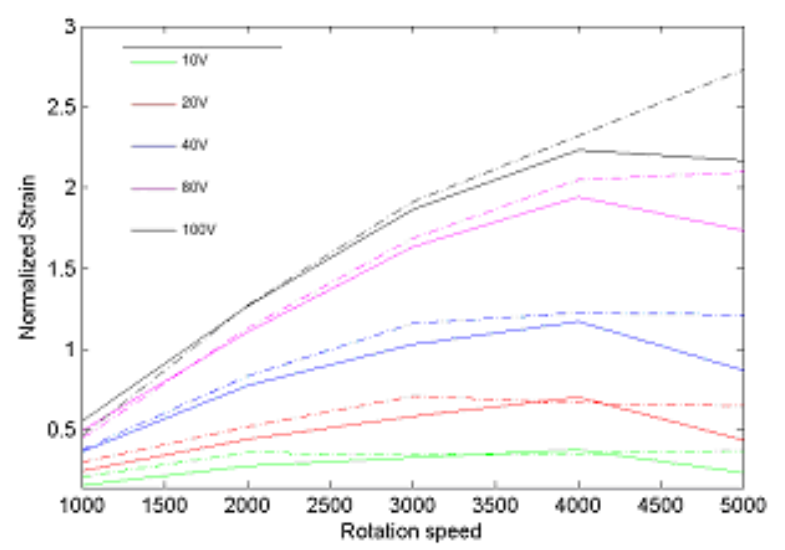

Figure 15 : Normalised strain at resonance for various excitation levels and various speeds. Experimental (solid line) and simulation (dashed line) results - Free dovetail joint without damper

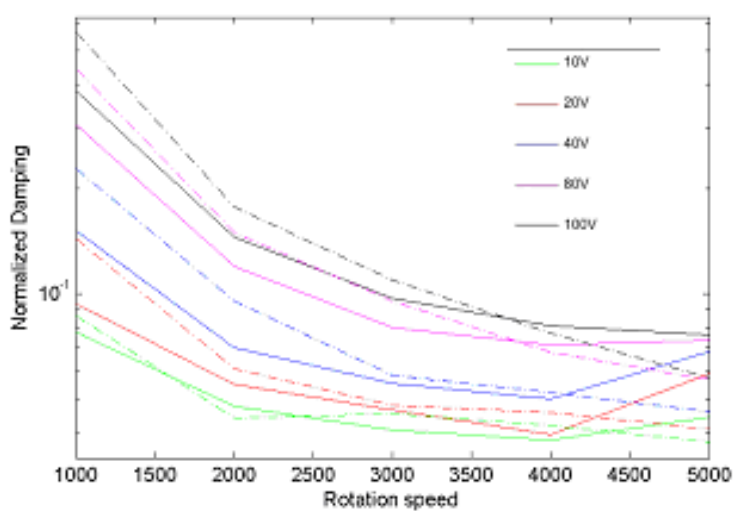

Figure 16 : Normalised equivalent damping for various excitation levels and various speeds. Experimental (solid line) and simulation (dashed line) results - Free dovetail joint without damper 


\section{Damper friction}

Here, two parameters of the model can be updated to approach the experimental results: the friction coefficient $\mu$ and the damper stiffness $\mathrm{k}$. It was chosen to update both parameters simultaneously for the entire excitation level and speed ranges, which enables to fit the experimental response satisfactorily, in term of strain amplitude (less than 2\% error) and resonance frequency, as it can be seen in Figure 17 and Figure 18 for 1000 and $4000 \mathrm{rpm}$ respectively. The shapes of the frequency responses found in simulation are "stiffer" than in the experiment, in so far as the damper state changes sharply when the frequency is varying around the resonance. Nearby the resonance frequency, the damper is in stick-slip alternance, which leads to a flat frequency response, whereas further from the resonance, the damper is in stick state.

The updated parameters $\mu$ and $\mathrm{k}$ are shown in Figure 19 and Figure 20. $\mu$ enables to adjust the amplitude and $\mathrm{k}$ the resonance frequency. The physical meaning of updated parameters can be questioned: $\mu$ and $\mathrm{k}$ reach very low values, compared to the static friction coefficient of steel-steel contact, and the real stiffness of the damper. Moreover, this update process is very dependant of the experiment, and it lacks of predictiveness, contrary to the DLFT method used in dovetail joint configuration. This is probably due to the fact that the DLFT method retains much more interface nodes (64 here) than the friction damper model (only 2 nodes). The next version of the damper model, which is currently being integrated at Snecma and will be soon used to reproduce the experiment considered here, enables to retain much more nodes, which should improve the predictiveness of the model.

With this update process though, the simulation is able to capture quite well the amplitude decrease and the frequency shift due to the presence of the friction damper.

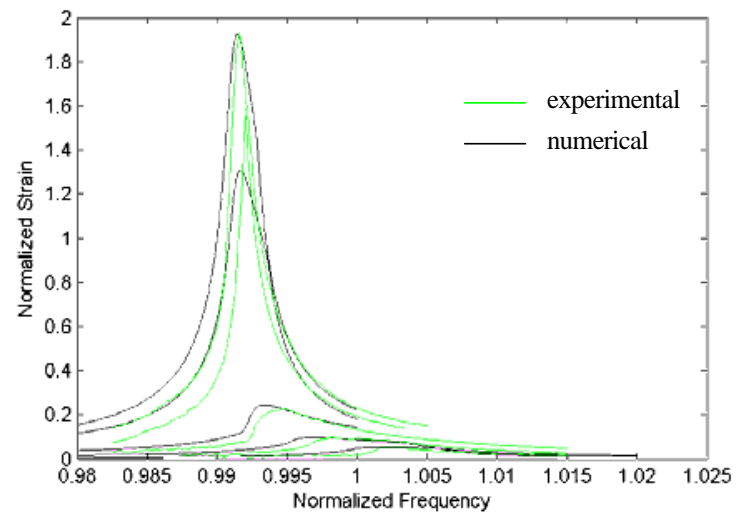

Figure 17 : Normalised experimental and numerical strain results for bonded dovetail joint with damper configuration, at $1000 \mathrm{rpm}$

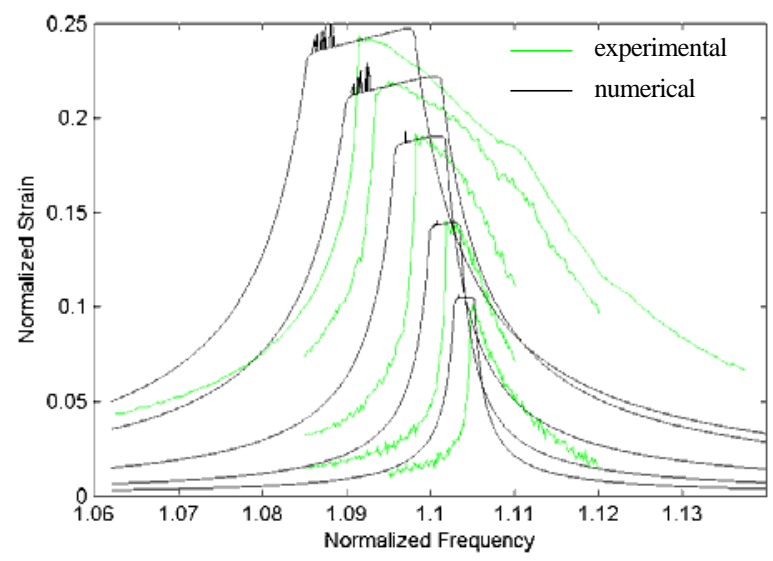

Figure 18 : Normalised experimental and numerical strain results for bonded dovetail joint with damper configuration, at $4000 \mathrm{rpm}$

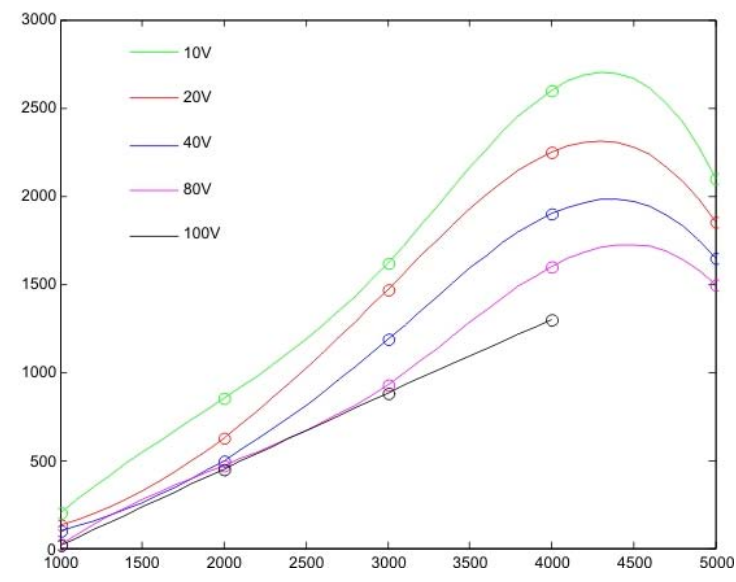

Figure 19 : Updated damper stiffness for various speeds and various excitations levels

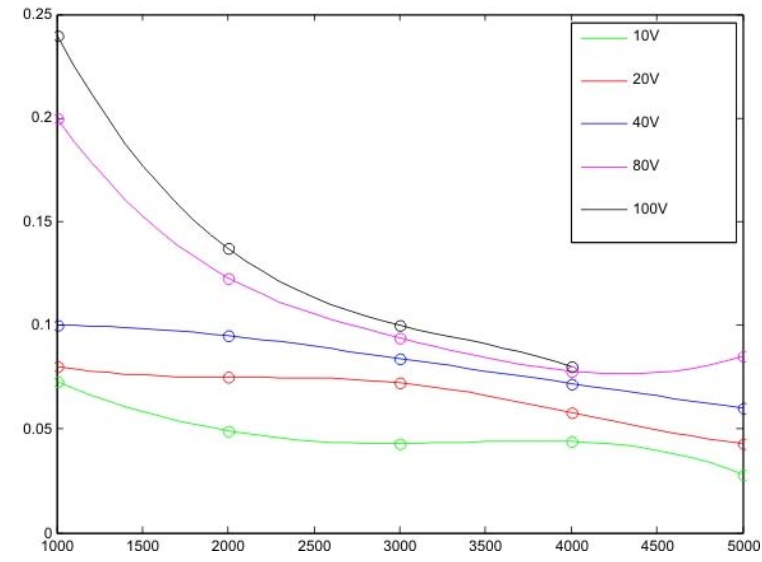

Figure 20 : Updated damper friction coefficient for various speeds and various excitations levels 


\section{CONCLUDING REMARKS}

The forced response of bladed disks in rotating conditions has been studied both experimentally and numerically, with 2 kinds of friction phenomena: contact at the blade-disk interface and under platform friction damper. The test rig and the experimental setup are representative of a real bladed-disk configuration. They have been considerably improved compared to previous campaigns in order to accentuate friction phenomena, to decouple them, and to have a linear reference for calibration of the models. For this, the blade root has been softened and some tests with bonded dovetail joints have been done.

The experimental disk has been modeled by a sector in cyclic symmetry to qualify two numerical tools of dynamic friction modeling, based on multiharmonic balance method. The first one, called DLFT, enabled to predict quite satisfactorily the experimental results with a unique value of friction coefficient for all the experimental conditions, which shows a certain performance and robustness of the method. The predictiveness of the model could be probably improved by implementing other friction laws, smoother than Coulomb's law, but also by modeling more precisely the piezoelectric exciter or increasing the number of retained nodes. The second tool, used to model the friction damper, retains at the moment only two nodes, which makes it less predictive, since it was needed to update the friction coefficient and the damper stiffness for each rotation speed and excitation level. Although the results are globally close to the experiment, an improved version of this tool with multiple contact points will be soon implemented. This tool should be more predictive and robust, which is necessary for an industrial use on new designs without any experimental reference.

\section{ACKNOWLEDGMENTS}

This work takes place in the framework of the MAIA mechanical research and technology program sponsored by CNRS, ONERA and SAFRAN Group.

\section{REFERENCES}

[1] Griffin, J.H., 1980, "Friction Damping of Resonant Stresses in Gas Turbine Engine Airfoils," ASME Journal of Engineering for Power, 102, pp. 329-333.

[2] Poudou, O., 2007 "Modeling and analysis of the dynamics of dry-friction-damped structural systems", $P h D$ thesis, University of Michigan.

[3] Charleux, D., 2006 "Numerical and Experimental Study of Friction Damping in Blade Attachments", PhD thesis, Ecole Centrale de Lyon.

[4] Seinturier, E., Lombard, J.P., Dumas, M., Dupont, C., Sharma, V., Dupeux, J., 2004, "Forced Response Prediction Methodology for the Design of HP Compressors Bladed Disks," proceedings ASME TurboExpo Paper Number GT2004-53372.

[5] Berthillier, M., Dupont, C., Mondal, R., Barrau, J.J., 1998, "Blades Forced Response Analysis with Friction Dampers," ASME Journal of Vibration and Acoustics, 120, pp. 468-474.

[6] Petrov, E. P., and Ewins, D. J., 2003. "Analytical formulation of friction interface elements for analysis of nonlinear multiharmonic vibrations of bladed disks". Journal of turbomachinery, 125, pp. 364-371.

[7] Poudou, O. Pierre, C., 2003, "Hybrid Frequency-Time Domain Methods for the Analysis of Complex Structural Systems with Dry Friction Damping," Collection of Technical Papers - AIAA/ASME/ASCE/AHS/ASC Structures, Structural Dynamics and Materials Conference, 1, pp. 111-124.

[8] Nacivet, S., Pierre, C., Thouverez, F., and Jezequel, L., 2003. "A dynamic Lagrangian frequency-time method for the vibration of dry-friction-damped systems". Journal of Sound and Vibration, 265, pp. 201-219.

[9] Charleux D., Gibert C., Thouverez F., and Dupeux J., 2006, "Numerical and Experimental Study of Friction Damping Blade Attachments of Rotating Bladed Disks," International Journal of Rotating Machinery, vol. 2006, Article ID 71302, 13 pages.

[10] Sever, I. A., Petrov, E. P., and Ewins, D. J., 2007 "Experimental and numerical investigation of rotating bladed disk forced response using under-platform friction damper", proceedings ASME TurboExpo, Montreal Canada, Paper Number GT2007-27307

[11] Szwedowicz J., Gibert C., Sommer T. P. and Kellerer R., 2006 "Numerical and experimental damping assessment of a thin-walled friction damper in the rotating setup with high pressure turbine blades", proceedings ASME TurboExpo, Barcelona, Spain, Paper Number GT2006-90951

[12] Craig, R.R., Bampton, M.C.C., 1968, "Coupling of Substructures for Dynamic Analysis", AIAA Journal, 6(7), pp. 1313-1319.

[13] York, A., 2005, "Experimental investigation of ratedependent inner hysteresis loops in PZT, PhD thesis, North Carolina State University 